\title{
ORIGINAL
}

\section{DIFERENCIAS EN EL NÚMERO DE CESÁREAS EN LOS PARTOS QUE COMIENZAN ESPONTÁNEAMENTE Y EN LOS INDUCIDOS}

\author{
Antonio Hernández Martínez (1), Ana Isabel Pascual Pedreño (1), Ana Belén Baño Garnés (1), María del \\ Rocio Melero Jiménez (1) y Milagros Molina Alarcón (2).
}

(1) Hospital "Mancha-Centro". Alcázar de San Juan. Ciudad Real.

(2) Facultad de Enfermería. Universidad de Castilla-La Mancha. Albacete.

Los autores declaran la ausencia de ningún tipo de conflicto de intereses.

\section{RESUMEN}

Fundamentos: La inducción del parto (IDP) puede estar asociada a mayores complicaciones para la mujer gestante. El objetivo de este trabajo fue determinar las diferencias en el número de cesáreas entre partos espontáneos y partos inducidos.

Métodos: Cohortes históricas sobre un total de 841 inducciones y 2.534 partos espontáneos realizados entre 2009 y 2011 en el Hospital "Mancha-Centro" de Alcázar de San Juan. Se empleó análisis multivariante por medio de regresión logística binaria para control de la confusión.

Resultados: La prevalencia de IDP fue del 22,9\%, presentándose como indicaciones más frecuentes la rotura prematura de membranas (RPM) de más de 12 horas $(22,7 \%)$ y diabetes mellitus mal controlada con un $22,5 \%$. Se observó relación entre inducción y riesgo de cesárea tanto en mujeres nulíparas $(\mathrm{OR}=2,68$; Intervalo de confianza [IC] $95 \%$ : 2,15-3,34) como en multíparas (OR=2,10; [IC] 95\%:1,72-2,57). La indicación con mayor riesgo de cesárea fue la gestación cronológicamente prolongada (GCP) $(37,1 \%)$ seguida del registro cardiotográfico (RCTG) patológico $(35,3 \%)$ y estados hipertensivos del embarazo (EHE) $(4,0 \%)$ La IDP también se relacionó con la mayor duración de la dilatación $(\mathrm{OR}=6,00$; IC 95\%:4,02-8,95), empleo de epidural (OR=3,10;IC95\%: $2,24-4,29)$ y necesidades de transfusión sanguínea $(\mathrm{OR}=3,33$; IC 95\%:1,70-9,67).

Conclusiones: La IDP es un factor de riesgo para una mayor duración de la dilatación, empleo de analgesia epidural, necesidad de transfusión sanguínea y de cesárea tanto en mujeres nulíparas como en multíparas, especialmente en las indicaciones de gestación cronológicamente prolongada, registro cardiotocográfico patológico y estados hipertensivos del embarazo. No se encontró relación con la duración del período expulsivo, la práctica de episiotomía, desgarros, sangrado excesivo y rotura uterina.

Palabras clave: Inicio del trabajo de parto. Trabajo de parto inducido. Cesárea. Complicaciones del trabajo de parto.

\section{Correspondencia}

Antonio Hernández Martínez

C/Gerona 25 Tomelloso

13700 Ciudad Real

Correo electrónico:antomatron@gmail.com

\section{ABSTRACT \\ Differences in Cesarean Sections between Spontaneous and Induced Labour}

Background: The induction of labour (IOL) may be associated with complications for pregnant women. The aim was to assess the differences in the number of caesarean sections between spontaneous or induced labour.

Methods: Historical groups from 841 women with induced labour and 2,534 women with an spontaneous onset of labour over a three-year period (from 2009 to 2011). They were carried out in "Mancha Centro" Hospital (Alcázar de San Juan). It was used a multivariate analysis through binary logistic regression to control confounding variables.

Results: The prevalence of IOL was $22,9 \%$. The most frequent indications were: Premature Rupture of Membranes (22,7\%), bad- controlled Diabetes $(22,5 \%)$. It was reported a relation between induced labour and cesarean section risk due to parity (nulliparous $\mathrm{OR}=2.68$, IC $95 \%: 2.15$ 3.34 and multiparous $\mathrm{OR}=2.10$, IC $95 \%: 1.72-2.57$ ). Postterm pregnancy $(37,1 \%)$, pathological monitor $(35.3 \%)$ and hypertensive diseases of pregnancy $(34 \%)$ reported the highest risks of cesarean section. The IOL was related to other factors: a long- time length first -stage of labour $(\mathrm{OR}=$ 6.00; IC 95\%: 4.02- 8.95), use of epidural analgesia (OR=3.10; IC 95\%: 2.24- 4.29) and blood transfusion needs $(\mathrm{OR}=3.33$; IC 95\%: 1.70- 9.67).

Conclusion: Independently of parity, The IOL increases the risk to: have a longer duration first- stage, use epidural analgesia, need a blood transfusion and have a cesarean section. This relation is stronger when induction is due to postterm pregnancy, pathological monitor or hypertensive diseases. No relation was found among induced labour and secondstage duration, episiotomy, perineal tears, excessive blood loss or uterine rupture.

Keyword: Labor onset. Labor induced. Caesarean section. Obstetric labor complications. 


\section{INTRODUCCIÓN}

La inducción del parto (IDP) no es una técnica en sí misma sino un conjunto de procedimientos médicos o mecánicos (rotura de membranas, administración de prostaglandinas y oxitócicos, etc) que tiene por objeto iniciar el trabajo de parto en una mujer gestante antes del momento establecido fisiológicamente ${ }^{1}$. La variabilidad con la que se emplea es alta, situándose en torno al $20 \%$ de todos los partos ${ }^{2,3}$, si bien en países como Estados Unidos supera en la actualidad el $30 \%{ }^{4}$. Además se observa una tendencia creciente en su empleo ${ }^{4-6}$.

Entre las indicaciones más frecuentes de IDP destacan las complicaciones médicas del embarazo (diabetes mellitus e hipertensión especialmente), rotura prematura de membranas (RPM), oligoamnios, gestación cronológicamente prolongada (GCP), etcétera. Sin embargo, también se contempla la posibilidad de inducción en ausencia de criterios médicos, lo que se conoce como inducción electiva. Esta indicación es poco relevante en la sanidad pública española, pero frecuente especialmente en el ámbito privado y en otros países ${ }^{8}$.

Uno de los inconvenientes de este tipo de parto, tanto en las indicaciones electivas como en las médicas, es el mayor riesgo de finalización por medio de cesárea. Estas mujeres reúnen por si mismas características que las colocan en desventaja frente a las que inician su parto de forma espontánea $^{6,8-13}$. Suelen ser madres o fetos con patologías o con alta probabilidad de que su estado de salud se vea comprometido si el embarazo se deja evolucionar naturalmente $\mathrm{y}$ con unas condiciones cervicales de mayor inmadurez. Una vez controlado el resto de variables, a la IDP se le atribuye el $20 \%$ de todas las cesáreas?.

Además, la inducción del parto se asocia a otras complicaciones obstétricas como atonía uterina, mayor empleo de analgesia, parto prolongado y laceraciones perineales $^{2,11,12}$. Se presenta, por tanto, una paradoja, pues se intenta, por un lado, desarrollar a nivel institucional estrategias para minimizar el número de cesáreas y otras complicaciones pero de forma paralela existe una mayor tendencia a inducir el parto ${ }^{1}$, lo que puede implicar en un aumento en la morbilidad materna y en la tasa de cesárea.

El objetivo del estudio es determinar las diferencias en los resultados obstétricos entre los partos con un inicio espontáneo y los inducidos.

\section{MATERIAL Y MÉTODOS}

Diseño. Estudio de cohortes retrospectivo, observacional, analítico. Se realizó en el servicio de partos del Complejo Hospitalario "Mancha-Centro" de Alcázar de San Juan (Ciudad Real) durante los años 2009, 2010 y 2011.

La población de referencia fue el conjunto de mujeres gestantes que recibieron asistencia en el parto. Se excluyó a las mujeres con cesáreas programadas, cesáreas urgentes no evitables (presentaciones podálicas, transversas, de frente y denegación de parto vaginal por cesárea anterior) y a las mujeres con gestaciones gemelares y muerte fetal anteparto.

Los criterios médicos para IDP en nuestro centro durante el periodo de estudio fueron:

- GCP : inducción en la semana 41+5 en base a la datación de la ecografía del primer trimestre.

- Oligoamnios: definido como un índice de líquido amniótico menor de 5.

- Hidramnios: definido como un índice de líquido amniótico superior a 25.

- Diabetes pregestacional: Si los controles fueron buenos se finalizó a las 39 semanas de gestación y si fueron malos (más de 
dos valores alterados en control semanal), se finalizó con más de 37 semanas, siempre que fue posible.

- Diabetes gestacional controlada con dieta: con buen control la IDP se hizo a las 41 semanas y con mal control a las 38 .

- Diabetes gestacional en tratamiento con insulina: con buen control la IDP se hizo a las 39 semanas y con mal control a las 38 .

- RPM de más de 12-24 horas de evolución.

- Líquido amniótico meconial tras amniorrexis espontánea o detectada por amnioscopia sistemática a partir de las 40 semanas.

- Estados hipertensivos del embarazo (EHE) con grave afectación materno-fetal o a partir de las 37 semanas en los casos de preeclampsia leve.

- Crecimiento intrauterino retardado (CIR): los tipo I (pequeños para edad gestacional con percetil $<3$ y doppler normal) y los tipo II (insuficiencia placentaria leve) se indujeron a las 37 semanas, los tipo III (insuficiencia placentaria severa) y en los tipo IV (hipoxia) y tipo V (signos de acidosis) se realizaron cesáreas programadas.

- Malos antecedentes obstétricos: cuando se produjo una muerte fetal anteparto en gestación anterior.

- La macrosomía y el hidramnios: aunque por sí solos no son criterio de inducción en nuestro centro, se detectaron como indicación en algunas ocasiones.

Para la elección del método de inducción se utilizó el índice de Bishop, escala de valoración de las condiciones cervicales con puntuaciones de 0 a 13 puntos:

- Si el índice de Bishop < 6: colocación de un dispositivo de liberación prolongada de dinoprostona $10 \mathrm{mgr}$ intravaginal durante
12 horas seguido de amniorrexis artificial y administración de oxitocina.

- Si índice de Bishop $\geq 6$ : amniorrexis e inducción oxitócica.

Los criterios médicos de finalización urgente de cesárea fueron los siguientes:

- El riesgo de perdida de bienestar fetal (RPBF) que englobó: acidosis fetal en $\mathrm{pH}$ intraútero $\mathrm{y} / \mathrm{o}$ ritmo sinusoidal $\mathrm{y} / \mathrm{o}$ bradicardia mantenida (por encima de 5-7 minutos) que no tuviera relación con hipertonía uterina o taquisistolia. También se incorporó en este criterio los eventos centinela asociados a sufrimiento fetal, como la rotura uterina, el prolapso de cordón, rotura de vasa previa, desprendimiento prematuro de placenta normoinserta (DPPNI).

- El fracaso de inducción (FI), definido como aquella IDP que tras 12 horas de dinámica uterina activa (2-3 contracciones de más de $40 \mathrm{mmHg}$ en 10 minutos) no se alcanzaron las condiciones establecidas de parto: cervix borrado, 2-3 cm de dilatación. En cesáreas anteriores el tiempo de espera fue de 9 horas.

- La no progresión del parto (NPP), definida como el parto en el que una vez establecidas las condiciones de trabajo activo transcurrieron más de 4 horas sin progresión de las condiciones obstétricas y la dilatación, con dinámica activa del parto y bolsa rota. En cesáreas anteriores se reducjo a 3 horas.

- La desproporción pélvico-fetal (DPC) establecida por los siguientes criterios: en dilatación completa, dinámica activa y pujos activos y el punto guía de la presentación sin pasar del tercer plano. Según paridad, existencia de cesárea anterior y empleo de epidural se consideraron los siguientes tiempos: mujeres sin analgesia epidural: 1 hora en multíparas, 2 horas en nulíparas y 1 hora en cesárea anterior. En mujeres con 
analgesia epidural: 2 horas en multíparas, 3 horas en primíparas y 1 hora y media en cesárea anterior. También se consideró la indicación de DPC cuando existió la imposibilidad de extraer el feto por vía vaginal tras una prueba de parto (instrumental fallida).

Fuentes de información. La información se recogió de las historias clínicas de las mujeres gestantes incluidas en el estudio.

Variables. De resultado principal: parto cesárea (si/no). Variables de resultado secundarias: indicación cesárea, empleo de analgesia epidural, sangrado excesivo (pérdida hemática $>3,5$ gramos de hemoglobina entre el inicio del parto y las primeras 48 horas postparto), rotura uterina, necesidad de transfusión, realización de histerectomía obstétrica, practica de episiotomía, desgarro perineal tipo III-IV, duración de la dilatación y duración del período expulsivo (ambos en minutos).

Variables independientes principales: inicio del parto (espontáneo/inducido) e indicación de inducción en parto iniciado.

Las variables de control fueron:edad materna, edad gestacional, cesárea anterior, paridad y peso del recién nacido.

Análisis estadístico. Se calcularon las frecuencias absolutas y relativas de las variables cualitativas y la media y su desviación estándar para las cuantitativas. Se realizó un análisis bivariante entre los factores de confusión con el inicio del parto (espontáneo/ inducido) empleando las pruebas de $\mathrm{chi}^{2}$ para variables cualitativas y $t$ de Student para las variables cuantitativas. Se desarrollaron diversos modelos multivariantes con regresión logística binaria para controlar el sesgo de confusión. Para el estudio de regresión logística se tuvo en cuenta la estrategia de modelización máxima, por la que la muestra debía tener 10 eventos (cesáreas) como mínimo por cada variable a analizar. Se estimaron Odds Ratio (OR) con sus respectivos intervalos de confianza del $95 \%$, empleando como categoría de referencia la opción más fisiológica o normal.

Para el análisis estadístico se utilizó el software PASW versión 19.0. (SPSS, Inc., Chicago, IL, USA).

Consideraciones éticas y legales. Se solicitó la aprobación de la comisión de investigación ético-clínica del centro, garantizando en todo momento la confidencialidad de las historias clínicas y su información.

\section{RESULTADOS}

La población de referencia estaba formada por 3.817 mujeres de las cuales se excluyeron $53(1,38 \%)$ por gestación gemelar, $13(0,34 \%)$ por muerte fetal anteparto, $307(8,04 \%)$ por cesárea programada y $83(2,17 \%)$ con indicación de cesárea programada pero realizadas de forma urgente, participando definitivamente $3.375(88,4 \%)$. De estas $2.534(75,1 \%)$ tuvieron un inicio de parto espontáneo y $841(24,9 \%)$ inducido.

La principal causa de inducción fue la RPM con un 191 (22,7\%), seguida por la diabetes (pregestacional y gestacional) mal controlada $189(22,5 \%)$ y el oligoamnios con un $136(16,2 \%)$. En la tabla 1 se presentan las indicaciones de inducción.

En el estudio de la relación entre el inicio del parto con las características obstétricas más importantes se observó que las mujeres con IDP presentaron con mayor frecuencia una edad igual o superior a 35 años $(23,5 \%$ vs $19,3 \%)$, una edad gestacional inferior a 37 semanas $(8,1 \%$ vs $4,5 \%)$ o igual o superior a 41 semanas $(21,4 \% v s$ $12,1 \%)$ y nuliparidad $(65,6 \%$ vs $50,3 \%)$. Por otro lado, no se observó relación estadísticamente significativa entre cesárea anterior y el inicio del parto (tabla 2). 
Tabla 1

\section{Tasa de indicaciones de inducción}

\begin{tabular}{|l|c|c|}
\hline Indicaciones de inducción & $\mathrm{n}$ & $\%$ \\
\hline Rotura prematura de membranas $>12$ horas & 191 & 22,7 \\
\hline Diabetes con malos controles & 189 & 22,5 \\
\hline Oligoamnios & 136 & 16,2 \\
\hline Embarazo cronológicamente prolongado & 116 & 13.8 \\
\hline Líquido amniótico meconial & 55 & 6,5 \\
\hline Estados hipertensivos del embarazo & 47 & 5,6 \\
\hline Sospecha de crecimiento intrauterino restringido & 37 & 4,4 \\
\hline Registro cardiotocográfico patológico & 17 & 2,0 \\
\hline Interés fetal /malos antecedentes & 13 & 1,5 \\
\hline Hidramnios & 12 & 1,4 \\
\hline Colestasis & 9 & 1,1 \\
\hline Metrorragia & 5 & 0,6 \\
\hline Electiva & 4 & 0,5 \\
\hline Macrosomía & 4 & 0,5 \\
\hline Presentación inestable & 3 & 0,4 \\
\hline Enfermedad materna & 1 & 0,1 \\
\hline Fiebre anteparto & 1 & 0,1 \\
\hline Missing & 1 & 0,1 \\
\hline Total & 841 & 100 \\
\hline
\end{tabular}

Del total de 3.375 partos a estudio $2.617(77,6 \%)$ fueron eutócicos, 222 $(6,6 \%)$ instrumentales y $535(15,9 \%)$ cesáreas. Los partos inducidos presentaron una OR de cesárea de 2,68 en el caso de mujeres nulíparas y de 2,10 para multíparas, frente a los partos espontáneos, tras ajustar por factores de confusión.

Asimismo en las mujeres con IDP se observó una OR de empleo de analgesia regional de 3,10 (IC95\%: 2,24-4,29), una OR de duración de la dilatación mayor o igual a 12 horas de 6,00 (IC95\%: 4,02$8,98)$ y una OR de necesidades de transfusión de 3,33 (IC95\%: 1,7-9,67) con respecto a los partos espontáneos. No se encontró relación estadísticamente significativa con el resto de variables a estudio. (tablas 3 y 4).

En la tabla 5 se muestran los datos del riesgo de cesárea según indicación.
Sobre la relación entre el inicio del parto con las indicaciones de cesárea resultó que la indicación más frecuente fue el RPBF para ambos grupos, siendo para partos espontáneos del 53,9\% (151) y para inducidos del 35,7\% (86) (tabla 6).

Sobre el riesgo de cesárea en función del motivo de inducción se encontró que entre las indicaciones con más de 10 casos, la GCP fue la que presentó un riesgo de cesárea del $37,1 \%$ y una OR de 4,59 veces mayor que un inicio espontáneo (IC95\%: 3,09-6,82), seguido del registro cardiotocográfico (RCTG) patológico con un 35,3\% (OR: 4,25; IC95\%: $1,56-11,59)$ y en tercer lugar los EHE con un 34,0\% (OR: 4,03; IC95\%: 2, 17-7,53).

Tabla 2

\section{Factores obstétricos y su relación con el inicio del parto}

\begin{tabular}{|l|c|c|c|}
\hline Variables & \multicolumn{3}{|c|}{ Inicio del parto } \\
\hline & $\begin{array}{c}\text { Espontaneo } \\
(\mathrm{n}=2534)\end{array}$ & $\begin{array}{c}\text { Inducido } \\
(\mathrm{n}=841)\end{array}$ & $\mathrm{p}$ \\
\hline Edad Materna & $80(3,2)$ & $26(3,1)$ & 0,035 \\
\hline$\leq 20$ años & $1956(77,5)$ & $617(73,5)$ & \\
\hline $25-35$ años & $487(19,3)$ & $197(23,5)$ & \\
\hline$\geq 35$ años & $2523(100)$ & $840(100)$ & \\
\hline Total & $113(4,5)$ & $68(8,1)$ & \\
\hline Edad gestacional & $2104(83,4)$ & $592(70,5)$ & \\
\hline$<37$ & $305(12,1)$ & $180(21,4)$ & \\
\hline $37-41$ & $2522(100)$ & $840(100)$ & \\
\hline$\geq 41$ & & & $<0,001$ \\
\hline Total & $1250(50,3)$ & $549(65,6)$ & \\
\hline Paridad & $1233(49,7)$ & $288(34,4)$ & \\
\hline Nulíparas & $2483(100)$ & $837(100)$ & \\
\hline Multíparas & $2336(94,0)$ & $796(95,1)$ & \\
\hline Total & $148(6,0)$ & $41(4,9)$ & \\
\hline Cesárea anterior & & $837(100)$ & \\
\hline Si & & & 0,252 \\
\hline No & & & \\
\hline Total & & & \\
\hline
\end{tabular}


Tabla 3

Riesgo de cesárea y su relación con el inicio del parto

\begin{tabular}{|c|c|c|c|c|c|c|}
\hline \multirow{3}{*}{ Variables } & \multicolumn{6}{|c|}{ Inicio del parto } \\
\hline & \multirow[b]{2}{*}{$\begin{array}{l}\text { Espontáneo } \\
(\mathrm{n}=2534)\end{array}$} & \multirow[b]{2}{*}{$\begin{array}{c}\text { Inducido } \\
(\mathrm{n}=841)\end{array}$} & \multicolumn{2}{|c|}{ Análisis Univariante } & \multicolumn{2}{|c|}{ Análisis Multivariante } \\
\hline & & & $\begin{array}{l}\text { OR (IC 95\%) } \\
\text { sin ajustar }\end{array}$ & Valor P & $\begin{array}{l}\text { OR (IC 95\%) } \\
\text { ajustada }\end{array}$ & $\mathrm{p}$ \\
\hline \multicolumn{7}{|c|}{ Modo de parto global $^{\mathrm{a}}$} \\
\hline Vaginal & $2246(88,6)$ & $594(70,6)$ & 1 & & 1 & \\
\hline Cesárea urgente & $288(11,4)$ & $247(29,4)$ & $3,24(2,67-3,93)$ & $<0,001$ & $2,41(1,96-, 98)$ & $<0,001$ \\
\hline Total & $2534(100)$ & $841(100)$ & & & & \\
\hline \multicolumn{7}{|c|}{ Modo parto. Nulíparas ${ }^{\mathrm{b}}$} \\
\hline Vaginal & $1045(83,6)$ & $337(61,4)$ & 1 & & 1 & \\
\hline Cesárea urgente & $205(16,4)$ & $212(38,6)$ & $3,21(2,55-4,03)$ & $<0,001$ & $2,68(2,15-3,34)$ & $<0,001$ \\
\hline Total & $1250(100)$ & $549(100)$ & & & & \\
\hline \multicolumn{7}{|c|}{ Modo parto. Multíparas ${ }^{\mathrm{b}}$} \\
\hline Vaginal & $1156(93,8)$ & $254(88,2)$ & 1 & & 1 & \\
\hline Cesárea urgente & $77(6,2)$ & $34(11,8)$ & $2,01(1,31-3,08)$ & 0,026 & $2,10(1,72-2,57)$ & 0,001 \\
\hline Total & $1233(100)$ & $288(100)$ & & & & \\
\hline
\end{tabular}

* Se ajustó por paridad, edad materna, edad gestacional, empleo de analgesia epidural, peso del recién nacido y cesárea anterior. ${ }^{\dagger}$ Se ajustó por edad materna, edad gestacional, empleo de analgesia epidural, peso del recién nacido y cesárea anterior.

Tabla 4

Resultados obstétricos y su relación con el inicio del parto

\begin{tabular}{|c|c|c|c|c|c|c|}
\hline \multirow{3}{*}{ Variables } & \multicolumn{6}{|c|}{ Inicio del parto } \\
\hline & & & \multicolumn{2}{|c|}{ Análisis univariante } & \multicolumn{2}{|c|}{ Análisis multivariante } \\
\hline & $\begin{array}{c}\text { Espontáneo } \\
(\mathrm{n}=.2534)\end{array}$ & $\begin{array}{c}\text { Inducido } \\
(\mathrm{n}=841)\end{array}$ & $\begin{array}{c}\text { OR (IC 95\%) } \\
\text { sin ajustar }\end{array}$ & $\mathrm{p}$ & $\begin{array}{l}\text { OR (IC 95\%) } \\
\text { ajustada }\end{array}$ & $\mathrm{p}$ \\
\hline Empleo epidural $^{*}$ & & & & $<0,001$ & & $<0,001$ \\
\hline No & $433(17,3 \%)$ & $47(5,7 \%)$ & 1 & & 1 & \\
\hline $\mathrm{Si}$ & $2072(82,7 \%)$ & $779(94,3 \%)$ & $3,46(2,53-4,73)$ & & $3,10(2,24-4,29)$ & \\
\hline Duración dilatación $^{\dagger}$ & & & & $<0,001$ & & $<0,001$ \\
\hline$<12$ horas & $2389(91,9 \%)$ & $734(88,9 \%)$ & 1 & & 1 & \\
\hline$\geq 12$ horas & $41(1,7 \%)$ & $92(11,1 \%)$ & $7,30(5,01-10,65)$ & & $6,00(4,02-8,95)$ & \\
\hline Duración expulsivo $^{\dagger}$ & & & & 0,808 & & 0,179 \\
\hline$<3$ horas & $2088(91,9 \%)$ & $564(91,6 \%)$ & 1 & & 1 & \\
\hline$\geq 3$ horas & $185(8,1 \%)$ & $52(8,4 \%)$ & $1,04(0,75-1,44)$ & & $0,79(0,56-1,11)$ & \\
\hline Practica episiotomía ${ }^{\dagger}$ & & & & 0,011 & & 0,623 \\
\hline No & $1387(62,0 \%)$ & $334(56,3 \%)$ & 1 & & 1 & \\
\hline $\mathrm{Si}$ & $849(38,0 \%)$ & $259(43,7 \%)$ & $1,26(1,05-1,52)$ & & $1,05(0,85-1,31)$ & \\
\hline Desgarro tipo III-IV ${ }^{\dagger}$ & & & & 1,000 & & 0,753 \\
\hline No & $2222(99,4 \%)$ & $590(99,5 \%)$ & 1 & & 1 & \\
\hline $\mathrm{Si}$ & $14(0,6 \%)$ & $3(0,5 \%)$ & $0,80(0,23-2,82)$ & & $0,81(0,23-2,95)$ & \\
\hline Sangrado excesivo & & & & 0,742 & & 0,646 \\
\hline$<3,5 \mathrm{gr} / 1 \mathrm{Hb}$ & $2177(92,1 \%)$ & $724(91,8 \%)$ & 1 & & 1 & \\
\hline$\geq 3,5 \mathrm{gr} / 1 \mathrm{Hb}$ & $186(7,9 \%)$ & $65(8,2 \%)$ & $1,05(0,78-1,41)$ & & $0,93(0,67-1,28)$ & \\
\hline Transfusiónc & & & & 0,001 & & 0,008 \\
\hline No & $2525(99,6 \%)$ & $829(98,6 \%)$ & 1 & & 1 & \\
\hline $\mathrm{Si}$ & $9(0,4 \%)$ & $12(1,4 \%)$ & $4,06(1,7-9,67)$ & & $3,33(1,7-9,67)$ & \\
\hline Rotura uterina ${ }^{\ddagger}$ & & & & 0,076 & & \\
\hline No & $2512(99,6 \%)$ & $839(100 \%)$ & & & & \\
\hline $\mathrm{Si}$ & $11(0,4 \%)$ & $0(0,00 \%)$ & & & & \\
\hline
\end{tabular}

* Se ajustó por paridad, edad materna, edad gestacional, peso del recién nacido y cesárea anterior. ${ }^{\dagger}$ Se ajustó por paridad, edad materna, edad gestacional, peso del recién nacido. cesárea anterior y empleo de analgesia epidural. * Se ajustó por paridad, edad materna, edad gestacional, peso del recién nacido, cesárea anterior, empleo de analgesia epidural y tipo de parto. 
Tabla 5

Riesgo de cesárea según indicación de inducción

\begin{tabular}{|l|c|c|c|c|}
\hline & \multicolumn{4}{|c|}{ Resultado del parto } \\
\hline Indicaciones & Vaginal & Cesárea & OR (IC 95\%) & $p$ \\
\hline RPM $>12$ horas & $136(71,2 \%)$ & $55(28,8 \%)$ & $3,15(2,25-4,42)$ & $<0,001$ \\
\hline Diabetes & $138(73,0 \%)$ & $51(27,0 \%)$ & $2,88(2,04-4,06)$ & $<0,001$ \\
\hline Oligoamnios & $102(75,0 \%)$ & $34(25,0 \%)$ & $2,60(1,73-3,91)$ & $<0,001$ \\
\hline GCP & $73(62,9 \%)$ & $43(37,1 \%)$ & $4,59(3,09-6,82)$ & $<0,001$ \\
\hline LA meconial & $37(67,3 \%)$ & $18(32,7 \%)$ & $3,79(2,13-6,75)$ & $<0,001$ \\
\hline EHE & $31(66,0 \%)$ & $16(34,0 \%)$ & $4,03(2,17-7,45)$ & $<0,001$ \\
\hline SCIR & $25(67,6 \%)$ & $12(32,4 \%)$ & $3,74(1,86-7,53)$ & $<0,001$ \\
\hline Registro cardiotográfico patológico & $11(64,7 \%)$ & $6(35,3 \%)$ & $4,25(1,56-11,59)$ & 0,001 \\
\hline Interés fetal /malos antecedentes & $10(76,9 \%)$ & $3(23,1 \%)$ & $2,33(0,64-8,55)$ & 0,186 \\
\hline Hidramnios & $10(83,3 \%)$ & $2(16,7 \%)$ & $1,56(0,34-7,15)$ & 0,564 \\
\hline Total & $573(70,5 \%)$ & $240(29,5 \%)$ & & \\
\hline
\end{tabular}

La OR se calculó en relación al inicio de parto espontáneo (tabla 3). El análisis solo se ha calculado para aquellas indicaciones con más de 10 casos.

Tabla 6

Indicación de cesárea e inicio del parto

\begin{tabular}{|l|c|c|}
\hline \multirow{2}{*}{ Indicación de cesárea } & \multicolumn{2}{|c|}{ Inicio del parto } \\
\cline { 2 - 3 } & Espontáneo & Inducido \\
\hline $\begin{array}{l}\text { Riesgo de pérdida de } \\
\text { bienestar fetal (RPBF) }\end{array}$ & $151(53,9)$ & $86(35,7)$ \\
\hline $\begin{array}{l}\text { Desproporción } \\
\text { pélvico-cefálica (DPC) }\end{array}$ & $57(20,4)$ & $24(10,0)$ \\
\hline Fracaso de inducción (FI) & $0(0,0)$ & $56(23,2)$ \\
\hline $\begin{array}{l}\text { No progresión de parto } \\
\text { (NPP) }\end{array}$ & $72(25,7)$ & $75(31,1)$ \\
\hline Total & $280(100)$ & $241(100)$ \\
\hline
\end{tabular}

\section{DISCUSIÓN}

La prevalencia de la IDP en nuestro estudio presentó valores intermedios a los notificados por países latinoamericanos, con cifras que oscilan entre el $5 \mathrm{y}$ el $20 \%^{2}$ y países como Nueva Zelanda con un $29,1 \%{ }^{5}$ y Estados Unidos, donde puede llegar al $40 \%{ }^{14,15}$. La única información disponible sobre esta práctica en España fue aportada por la Comunidad Valenciana en el año 2010 con un tasa del $31,7 \%{ }^{16}$. A día de hoy se desconoce en el conjunto del Sistema Nacional de Salud Español el empleo de la IDP y sus implicaciones.
Respecto a las indicaciones hay que resaltar que nuestros resultados presentan discrepancias con los hallados en dos grandes estudios, el de Mealing et al. ${ }^{5}$ realizado en Australia, en el que la primera causa de inducción fue la GCP con un 33,4\%, seguido de los EHE con un 13,0\% y la RPM con un $11 \%$, y el de Guerra et al. ${ }^{2}$ realizado en 8 países latinoamericanos, donde la primera causa fue la inducción electiva seguida de la RPM y la GCP. En España se han realizado pocos trabajos sobre la inducción del parto presentándose la $\mathrm{RPM}^{17}$ y la $\mathrm{GCP}^{19}$ como las principales causas de inducción. Merece la pena destacar que indicaciones como el hidramnios y la macrosomía no son criterios de indicación de IDP del centro y sin embargo fueron empleados en algunas ocasiones. Entre los posibles motivos que puedan justificar estas diferencias tanto en la tasa global como por indicaciones se encuentran los diferentes protocolos de trabajo de cada centro y/o país, el peso de la medicina privada y la diferente presión legal a la que se ven sometidos los profesionales en cada ámbito.

En cuanto a las diferencias observadas entre las mujeres gestantes sometidas a 
inducción de parto y las que tienen un inicio de parto fue espontáneo, encontramos que hay una mayor proporción de nulíparas, gestaciones pretérmino, gestaciones prolongadas y madres de mayor edad dentro del grupo de inducción, coincidiendo en este punto con otros trabajos $2,5,6,11$. Esto pone de manifiesto que las gestaciones que requieren inducción parten, en general, en desventaja de cara a conseguir un parto eutócico. Todos estos factores están relacionados con un mayor grado de distocia ${ }^{8,20,21}$, independientemente de las condiciones cervicales y la presencia de alguna situación patológica.

En lo que se refiere al empleo de analgesia regional, se observó una mayor utilización en el grupo de las mujeres con IDP, hecho compartido con otros autores ${ }^{2,11}$, llegando a alcanzar en algún estudio una diferencia superior al $30 \%{ }^{12}$. Estas discrepancias pueden atribuirse a la distinta disponibilidad de recursos, protocolos de trabajo y demandas de las mujeres. En nuestro centro, uno de los motivos de mayor empleo se puede asociar a una mayor duración del periodo de dilatación en el grupo de IDP, siendo las dilataciones superiores a 12 horas de duración 6 veces más frecuentes en los partos inducidos. Estos resultados coinciden con el trabajo de Harper et al. ${ }^{22}$. Sin embargo, Cheng et al. ${ }^{23}$ no encontraron diferencias en la duración de la dilatación, hecho que puede explicarse porque estos autores no contabilizaron el tiempo que transcurrió hasta que las inducciones entraron en fase activa de parto.

Respecto a la duración del periodo expulsivo no se encontraron diferencias con expulsivos de más de tres horas entre ambos grupos. Esta ausencia de relación se justifica porque lo complejo de la inducción es llegar a la fase activa del parto, una vez alcanzado el periodo expulsivo el tipo de comienzo no debería condicionar su duración, coincidiendo con el trabajo de Myles et $\mathrm{al}^{24}$.

Por otro lado, no encontramos relación entre la tasa de episiotomías y desgarros perineales graves con el inicio del parto inducido. Esta ausencia de relación se ha observado en otros trabajos ${ }^{11,25}$, aunque no encontramos en la literatura nada publicado sobre la influencia de la IDP en la tasa de episiotomía.

En cuanto al sangrado durante el parto, observamos que no existen diferencias en cuanto a una pérdida igual o mayor a $3,5 \mathrm{gr} / 1$ de hemoglobina pero sí un aumento de las necesidades de transfusión en la inducción. Es decir, el porcentaje de sangrado excesivo es similar en ambos grupos pero los sangrados más graves que requirieron transfusión se concentraron en el grupo de inducción. Posiblemente es debido a un mayor grado de atonía producido a su vez por la mayor duración de la fase de dilatación. Existen varios trabajos que relacionan la inducción del parto con un mayor sangrado durante el parto $^{12,26}$.

Tampoco se observó asociación entre inducción y rotura uterina. Todos los casos de rotura se registraron en el grupo de parto espontáneo. Al tratarse de un evento raro, con nuestro tamaño muestral no hubo la suficiente potencia estadística para establecer conclusiones claras al respecto. Si bien, en un estudio realizado en Holanda sobre 371.021 partos se encontró un riesgo relativo de 3,6 con respecto a los espontáneos, aunque con un riesgo absoluto global bajo (1 por cada 1.708 partos) ${ }^{28}$. El riesgo de rotura parece estar especialmente relacionado con el empleo de prostaglandinas E1 (misoprostol) diferentes a las empleadas es este estudio (PGE2) y en gestantes con cesárea anterior ${ }^{29}$.

En lo que se refiere al riesgo de cesárea según el inicio del parto, encontramos una OR global de cesárea de 2,4 veces mayor en el grupo de IDP sobre los inicios espontáneos, una vez controlados los posibles factores de confusión, coincidiendo con diversos trabajos, que encuentran que los partos induci- 
dos presentan el doble de riesgo de cesárea que los espontáneos 10,13,14. También coincidimos con la literatura en el caso de las mujeres nulíparas, observándose un riesgo de cesárea entre 2 y 3 veces superior que los inicios espontáneos ${ }^{6,9,12,30}$. En el caso de las mujeres multíparas también encontramos un aumento de este riesgo. Sin embargo, si bien diversas publicaciones encuentran resultados similares al nuestro ${ }^{10,14,30}$, existe algún trabajo donde no se observó riesgo aumentando con respecto a los espontáneos ${ }^{11}$.

Por tanto, una de las medidas clave para reducir el número de cesáreas pasa un por una reducción del número de IDP, en este sentido algunas sociedades científicas como la Canadiense considera útil la creación de comités para reducir y ajustar el número de indicaciones de inducción ${ }^{29}$.

Por otro lado, se estudiaron los diferentes tipos de indicación de cesárea encontrando que el RPBF se presentó como la primera indicación tanto en espontáneos como inducidos. En otras publicaciones, esta indicación se sitúa entre el 27,3\% y el 47,2\% de todas las indicaciones de cesárea ${ }^{12,14}$ y podría ser la primera causa si no fuera porque estos trabajos agrupan la NPP, la DPC y el fracaso de inducción en una sola entidad.

Respecto al riesgo de cesárea según la indicación de la inducción, observamos que la indicación que presenta una mayor probabilidad de cesárea es la GCP, seguida del RCTG patológico y los EHE frente al inicio espontaneo.

Después de todo lo expuesto, cabe destacar como puntos fuertes de este trabajo un tamaño muestral adecuado y un escaso número de pérdidas. Además, el empleo de análisis multivariante, ausente en otros trabajos ${ }^{6,12,15}$, permite controlar el sesgo de confusión y conocer de forma más clara las diferencias en la evolución y resultados obstétricos de un proceso tan frecuen- te y tan sujeto a variabilidad como es la inducción de parto.

En cuanto a los puntos débiles, debemos ser conscientes de que los resultados obstétricos que se obtienen dependen en gran medida de la variabilidad de las indicaciones de inducción, de los métodos de IDP empleados, así como de los criterios médicos de indicación de cesárea. A consecuencia de esto, surge la principal limitación del trabajo, puesto que las complicaciones y resultados observados en el parto pueden variar considerablemente en cada centro. Asimismo, otra limitación observada fue la falta de registro del índice de masa corporal, siendo este un factor relacionado con el riesgo de cesárea ${ }^{31}$, aunque pensamos que modificaría poco los resultados finales.

Como implicaciones para la investigación consideramos que este estudio es de los pocos, concretamente en el ámbito español, que aporta información detallada sobre el comportamiento de los partos inducidos frente a los espontáneos y especialmente determina el riesgo de cesárea en función del tipo de indicación, además de consolidar determinados factores como responsables de peores resultados obstétricos.

Los resultados observados de cara a la práctica clínica pueden servir para que el profesional disponga de más información sobre los principales resultados asociados a inducción de parto, así como las posibilidades de parto en función de la indicación de inducción.

Sería recomendable reproducir este trabajo en otros ámbitos del Sistema Nacional de Salud para conocer la realidad de la inducción de parto, sirviendo de base para la puesta en marcha de una nueva estrategia y la creación de guías de práctica clínica, al igual que en el caso del parto normal para establecer estándares de resultados obstétricos en función del tipo de indicación de IDP y reducir la variabilidad en este campo. 
Podemos concluir que la IDP es un factor de riesgo para una mayor duración del período de dilatación, empleo de analgesia epidural, necesidad de transfusión sanguínea y de cesárea tanto en mujeres nulíparas como en multíparas, especialmente en las indicaciones de GCP, RCTG patológico y EHE.

\section{AGRADECIMIENTOS}

El equipo de investigación quiere agradecer a los profesionales del servicio de Archivo del centro por su colaboración y al Dr. Tenias por sus aportaciones metodológicas y revisión del manuscrito.

\section{BIBLIOGRAFÍA}

1. Sociedad Española de Ginecologia y Obstetricia. Induccion de parto. Protocolos prosego [citado 15 de dic. 2013]. Disponible en: http://www.prosego.es

2. Guerra GV, Cecatti JG, Souza JP, Faundes A, Morais SS, Gulmezoglu AM et al. Factors and outcomes associated with the induction of labour in Latin America. BJOG. 2009;116:1762-72.

3. Humphrey T, Tucker JS. Rising rates of obstetric interventions: exploring the determinants of induction of labour. J Public Health (Oxf). 2009;31:88-94.

4. Zhang X, Joseph KS, Kramer MS. Decreased term and postterm birthweight in the United States: impact of labor induction. Am $\mathrm{J}$ Obstet Gynecol. 2010;203:124-7.

5. Mealing NM, Roberts CL, Ford JB, Simpson JM, Morris JM. Trends in induction of labour, 1998-2007: a population-based study. Aust N Z J Obstet Gynaecol. 2009 ;49:599-605.

6. Patterson JA, Roberts CL, Ford JB, Morris JM. Trends and outcomes of induction of labour among nullipara at term. Aust $\mathrm{N}$ Z J Obstet Gynaecol 2011;51:510-7

7. Grupo de variaciones en la práctica médica. Variaciones en la utilización de la cesárea en los hospitales públicos del Sistema Nacional de Salud. Documento de trabajo 04-2009 [citado 15 de dic. 2013]. Disponible en: http://www.asturias.es/Astursalud/Ficheros/AS Salud $\% 20$ Publica/AS Promocion $\% 20$ de $\% 201 \mathrm{a} \% 20 \mathrm{Sa}$ lud/Salud $\% 20$ sexual $\% 20 y \% 20$ reproductiva/Salud $\% 20$ r eproductiva/Variaciones\%20cesarea_DT04_2009.pdf
8. Cnattingius R, Hoglund B, Kieler H. Emergency cesarean delivery in induction of labor: an evaluation of risk factors. Acta Obstet Gynecol Scand. 2005;84:456-62.

9. Ehrenthal DB, Jiang X, Strobino DM. Labor induction and the risk of a cesarean delivery among nulliparous women at term. Obstet Gynecol. 2010;116:35-42.

10. Glantz JC. Term labor induction compared with expectant management. Obstet Gynecol. 2010;115:70-6.

11. Janakiraman V, Ecker J, Kaimal AJ. Comparing the second stage in induced and spontaneous labor. Obstet Gynecol. 2010;116:606-11.

12. Selo-Ojeme D, Rogers C, Mohanty A, Zaidi N, Villar R, Shangaris P. Is induced labour in the nullipara associated with more maternal and perinatal morbidity? Arch Gynecol Obstet. 2011;284:337-41.

13. Thorsell M, Lyrenas S, Andolf E, Kaijser M. Induction of labor and the risk for emergency cesarean section in nulliparous and multiparous women. Acta Obstet Gynecol Scand. 2011;90:1094-9.

14. Zhang J, Troendle J, Reddy UM, Laughon SK, Branch DW, Burkman R, et al. Contemporary cesarean delivery practice in the United States. Am J Obstet Gynecol. 2010;203:326.

15. Laughon SK, Zhang J, Grewal J, Sundaram R, Beaver J, Reddy UM, et al. Induction of labor in a contemporary obstetric cohort. Am J Obstet Gynecol. 2012;206:486-9.

16. Health and care of pregnant women and babies in europe in 2010. European perinatal health report [citado 15 de dic. 2013]. Disponible en: http://www.europeristat.com/reports/european-perinatal-health-report-2010.html

17. Mozurkewich E, Chilimigras J, Koepke E, Keeton K, King VJ. Indications for induction of labour: a best-evidence review. BJOG. 2009;116:626-36.

18. Luengo A, Zornoza V, Fernandez-Coro A, et al. Indice de masa corporal y aumento de peso en el embarazo. Resultado obstétrico de la inducción de parto. Clin Invest Gin Obst. 2012;39:199-202.

19. San Frutos L, Bueno B, Engels V, Perez-Medina T, Barbancho C, Salazar F, et al. Induccion del parto: variables clínicas que influyen hasta el inicio de la fase activa del parto. Prog Obstet Ginecol. 2005;48:74-8 
20. Dulitzki M, Soriano D, Schiff E, Chetrit A, Mashiach S, Seidman DS, et al. Effect of very advanced maternal age on pregnancy outcome and rate of cesarean delivery. Obstet Gynecol. 1998;92:935-9.

21. Dyachenko A, Ciampi A, Fahey J, Mighty H, Oppenheimer L, Hamilton EF, et al. Prediction of risk for shoulder dystocia with neonatal injury. Am J Obstet Gynecol. 2006 ;195:1544-9.

22. Harper LM, Caughey AB, Odibo AO, Roehl KA, Zhao Q, Cahill AG. Normal progress of induced labor. Obstet Gynecol. 2012;119:1113-8.

23. Cheng YW, Delaney SS, Hopkins LM, Caughey AB. The association between the length of first stage of labor, mode of delivery, and perinatal outcomes in women undergoing induction of labor. Am J Obstet Gynecol. 2009;201:477.

24. Myles TD, Santolaya J. Maternal and neonatal outcomes in patients with a prolonged second stage of labor. Obstet Gynecol. 2003;102:52-8.

25. Battista L, Chung JH, Lagrew DC, Wing DA. Complications of labor induction among multiparous women in a community-based hospital system. Am J Obstet Gynecol. 2007;197:241-7.

26. Khireddine I, Le RC, Dupont C, Rudigoz RC, Bouvier-Colle MH, Deneux-Tharaux C. Induction of labor and risk of postpartum hemorrhage in low risk parturients. PLoS One. 2013;8:e54858.

27. Kramer MS, Dahhou M, Vallerand D, Liston R, Joseph KS. Risk factors for postpartum hemorrhage: can we explain the recent temporal increase? J Obstet Gynaecol Can. 2011;33:810-9.

28. Zwart JJ, Richters JM, Ory F, de Vries JI, Bloemenkamp KW, van RJ. Uterine rupture in The Netherlands: a nationwide population-based cohort study. BJOG. 2009;116:1069-78.

29. Leduc D, Biringer A, Lee L, Dy J, Corbett T, Leduc $\mathrm{D}$ et al. Induction of labour. J Obstet Gynaecol Can. 2013;35:840-57.

30. Vrouenraets FP, Roumen FJ, Dehing CJ, van den Akker ES, Aarts MJ, Scheve EJ. Bishop score and risk of cesarean delivery after induction of labor in nulliparous women. Obstet Gynecol. 2005;105:690-7.

31. Wolfe KB, Rossi RA, Warshak CR. The effect of maternal obesity on the rate of failed induction of labor. Am J Obstet Gynecol. 2011;205:128-7. 